\title{
Concomitant mitral regurgitation: an insidious cause of low- flow, low-gradient severe aortic stenosis
}

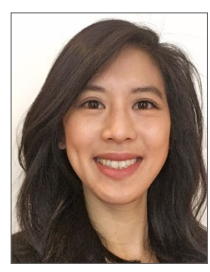

Géraldine Ong, MD; Marie-Annick Clavel, DVM, PhD; Philippe Pibarot*, DVM, PhD

Institut Universitaire de Cardiologie et de Pneumologie de Québec/Québec Heart and Lung Institute, Department of Medicine, Laval University, Québec, Canada

The combination of aortic stenosis (AS) and mitral regurgitation (MR) is frequent in patients with valvular heart disease. This entity raises important challenges with regard to severity grading and therapeutic management of both AS and MR ${ }^{1}$. Long-standing afterload excess associated with severe AS induces hypertrophic remodelling, dilatation and/or dysfunction of the left ventricle (LV) (Figure 1). Secondary MR may develop in this context as a result of mitral valve leaflet tethering and mitral annular dilatation. Because of the high prevalence of concomitant coronary artery disease, ischaemic MR is also frequent in the elderly population with AS. Elderly patients may also present primary MR as a result of degenerative and calcified mitral valves.

In the Euro Heart Survey, multiple valve disease, as defined by at least two moderate valvular diseases, was observed in $20 \%$ of the patients with native valve disease and in $17 \%$ of those undergoing intervention ${ }^{2}$. In a Swedish nationwide study, multiple and mixed valve diseases accounted for $11 \%$ of patients ${ }^{3}$ and the most frequent combinations were i) AS and MR, and ii) aortic regurgitation and MR. Despite the relatively high prevalence of the concomitance of $\mathrm{AS}$ and $\mathrm{MR}$, there are limited data on the pathophysiology, diagnosis, and management of this multiple valve disease entity.

\section{Underestimation of AS severity in the presence of MR}

In an in vitro study published in this issue of EuroIntervention, Katte et $\mathrm{al}^{4}$ provide an elegant demonstration of the strong interdependence between aortic valve and mitral valve haemodynamics.

\section{Article, see page 1635}

Using a pulsatile circulatory model, the authors investigated the effect of the presence and severity of MR on aortic valve area (AVA) and mean transvalvular gradient (MG) in different degrees of AS severity. In the presence of severe AS (AVA $\sim 0.5 \mathrm{~cm}^{2}$ ), the worsening of MR from none/mild to severe resulted in a marked decrease in the forward LV stroke volume (from 70 to $47 \mathrm{~mL}$ ) and thus in the MG (from 57 to $33 \mathrm{mmHg}$ ), whereas AVA remained unchanged. Hence, the backward flow induced by MR steals blood flow away from the aortic valve and therefore results in a low-flow state (forward stroke volume index $<35 \mathrm{~mL} / \mathrm{m}^{2}$ ) (Figure 1). Given that the MG is highly flow-dependent, the presence of a low-flow 
state may lead to a pseudo-normalisation of $\mathrm{MG}$ and therefore to an underestimation of AS severity. As a matter of fact, in the present $\mathrm{study}^{4}$, the induction of a severe MR brought the MG from very severe $(>50 \mathrm{mmHg})$ to moderate $(<40 \mathrm{mmHg})$ range, while the AVA remained severe $\left(<1.0 \mathrm{~cm}^{2}\right)$. This "discordant grading" situation with the concomitance of a small AVA $\left(<1.0 \mathrm{~cm}^{2}\right)$ and a low MG ( $<40 \mathrm{mmHg}$ ) may raise uncertainty about the true severity of the AS and thus about the therapeutic management of the patient in the clinical setting. In the light of the results of the present study by Katte et $\mathrm{al}^{4}$, there is clearly a potential to underestimate AS severity in the presence of $\geq$ moderate MR, if one relies only on MG or peak aortic jet velocity. The AVA is less affected by the low-flow state induced by MR and may be more reliable to assess true AS severity in the presence of MR. However, the AVA is also flow-dependent to some extent and it may be pseudo-severe.
There are three main types of discordant grading with small AVA and low $\mathrm{MG}^{5,6}$ : i) classic low-flow, low-gradient AS with reduced LV ejection fraction (LVEF), in which the low-flow state is mainly related to the depressed LV systolic function, ii) paradoxical low-flow, low-gradient AS with preserved LVEF, in which the low-flow state is often, but not always, due to pronounced LV concentric remodelling and impaired LV filling, and iii) normalflow, low-gradient AS (in this situation the LVEF and forward stroke volume index are within normal range but the MG may nevertheless be low despite the presence of a severe AS). Some studies suggest that reduced arterial compliance may be one of the explanations for the normal-flow low-gradient pattern ${ }^{5,7}$. The conclusion of Katte et $\mathrm{al}^{4}$ that arterial compliance does not alter aortic valve haemodynamics should be interpreted with caution given that reduced arterial compliance was associated with a 5 to

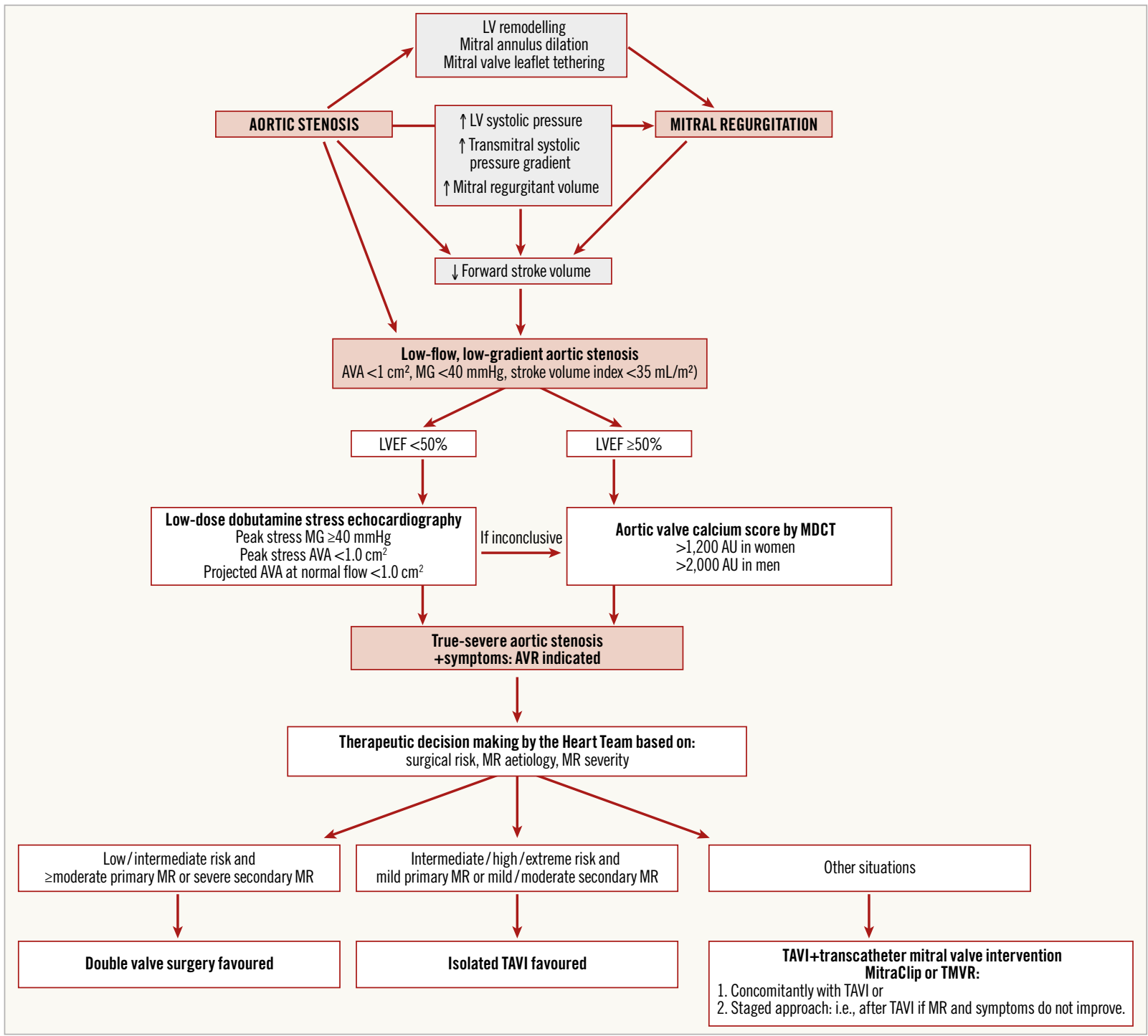

Figure 1. Pathophysiology, diagnosis, and therapeutic management of low-flow, low-gradient aortic stenosis associated with mitral regurgitation. AVA: aortic valve area; AVR: aortic valve replacement; LVEF: left ventricular ejection fraction; MG: mean transaortic gradient; MR: mitral regurgitation; TAVI: transcatheter aortic valve implantation 
$10 \mathrm{mmHg}$ reduction in MG in the most severe degrees of AS and that the standard deviations were relatively large.

The present study by Katte $\mathrm{al}^{4}$ underlines the point that concomitant moderate or severe MR may actually cause a paradoxical low-flow, low-gradient AS pattern. Indeed, MR may substantially reduce the forward LV stroke volume and thus the MG, despite a preserved LVEF. Benfari et al also reported that MR is an independent determinant of low-flow state in patients with severely reduced AVA and preserved $\mathrm{LVEF}^{8}$. Other potential causes of paradoxical low-flow, low-gradient AS include atrial fibrillation, mitral stenosis, pulmonary regurgitation, tricuspid regurgitation, constrictive pericarditis, and cardiac amyloidosis ${ }^{5,6}$. Concomitant MR is also frequent in patients with classic (low LVEF) low-flow, low-gradient AS and may contribute to the low transaortic flow state in these patients.

The interaction between aortic valve and mitral valve haemodynamics is bidirectional and AS may also alter the grading of MR severity. Indeed, the increased LV afterload caused by AS results in an increase of the transmitral systolic pressure gradient, therefore leading, for any given mitral effective regurgitant orifice, to higher mitral regurgitant volume (Figure 1$)^{1}$. Hence, in the presence of AS, there is a potential to overestimate the anatomic severity of MR when relying on regurgitant volume.

\section{Confirmation of AS severity in the presence of MR}

Regardless of the underlying aetiology and mechanism of the low-gradient AS pattern, the main clinical challenge, is to differentiate a true-severe from a pseudo-severe stenosis (Figure 1). The confirmation of stenosis severity is, indeed, crucial to guide therapeutic management in the patients with discordant (small AVA-low MG) grading. Low-dose dobutamine stress echocardiography may be used for this purpose in patients with low-flow, low-gradient $\mathrm{AS}^{5,6,9}$. However, in patients with concomitant MR, dobutamine stress may actually worsen the MR and fail to increase the forward flow across the aortic valve. Another approach to confirm AS severity in these patients is thus to quantitate aortic valve calcium load by multi-detector com-

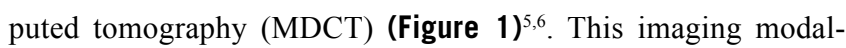
ity is probably the best option for patients with low-gradient AS and concomitant MR, and especially those with preserved LVEF (i.e., paradoxical low-flow, low-gradient AS). An aortic valve calcium score $>1,200$ in women and $>2,000$ AU in men is consistent with true-severe $\mathrm{AS}^{7,9}$.

\section{Therapeutic management of low-gradient AS with concomitant MR}

In the presence of low-gradient AS and concomitant MR, aortic valve replacement (AVR) is indicated if dobutamine stress echocardiography and/or MDCT confirm the presence of truesevere stenosis and the patient has symptoms and/or reduced LVEF (Figure 1) ${ }^{9}$. The next two key questions that need to be addressed by the Heart Team are: i) which type of AVR should be used in this context? and ii) should MR be corrected at the time of AVR or after AVR, or should it be left untreated? To answer these questions, it is important to take into account the patient's surgical risk as well as the aetiology and severity of the MR (Figure 1) ${ }^{1}$. The presence of moderate/severe MR is associated with increased risk of mortality and rehospitalisation following AVR, when the MR is left untreated ${ }^{10,11}$. Furthermore, secondary MR has a higher likelihood than primary MR to regress after AVR ${ }^{11}$. Hence, transcatheter aortic valve implantation (TAVI) should be preferred in patients with intermediate/high/extreme surgical risk and mild primary MR or mild/moderate secondary MR (Figure 1). On the other hand, double valve surgery should be favoured in patients with low/intermediate risk and severe secondary MR or moderate/ severe primary MR. In patients treated with TAVI, it is also possible to treat the MR concomitantly (i.e., at the time of TAVI) or as a staged approach (i.e., after TAVI if MR and symptoms do not improve) using the MitraClip ${ }^{\circledR}$ (Abbott Vascular, Santa Clara, CA, USA) or transcatheter mitral valve replacement (Figure 1). The staged approach should be considered particularly when the likelihood of MR regression after TAVI is uncertain ${ }^{1,11}$.

\section{Conclusions}

The coexistence of moderate/severe MR may lead to underestimation of AS severity, particularly when relying on MG or peak jet velocity to grade AS severity. On the other hand, moderate/severe AS may lead to overestimation of MR severity, particularly when relying on regurgitant volume. By reducing the forward flow across the aortic valve, concomitant MR may cause a paradoxical lowflow, low-gradient AS pattern. Particular attention should be paid to this insidious subtype of low-flow, low-gradient AS. Quantitation of aortic valve calcification by MDCT may help to corroborate the presence of true-severe AS and thereby confirm the indication of AVR in this situation. The decision making regarding the need for and type of procedure for the correction of AS and eventually MR should be individualised according to the patient's surgical risk, MR aetiology and MR severity (Figure 1).

\section{Conflict of interest statement}

The authors have no conflicts of interest to declare.

\section{References}

1. Unger P, Clavel MA, Lindman BR, Mathieu P, Pibarot P. Pathophysiology and management of multivalvular disease. Nature reviews. Cardiology. 2016;13:429-40.

2. Iung B, Baron G, Butchart EG, Delahaye F, GohlkeBarwolf C, Levang OW, Tornos P, Vanoverschelde JL, Vermeer F, Boersma E, Ravaud P, Vahanian A. A prospective survey of patients with valvular heart disease in Europe: The Euro Heart Survey on Valvular Heart Disease. Eur Heart J. 2003;24:1231-43.

3. Andell P, Li X, Martinsson A, Andersson C, Stagmo M, Zöller B, Sundquist K, Smith JG. Epidemiology of valvular heart disease in a Swedish nationwide hospital-based register study. Heart. 2017;103:1696-703. 
4. Katte F, Franz M, Jung C, Figulla HR, Leistner D, Jakob P, Staehli BE, Kretzschmar D, Lauten A. Impact of concomitant mitral regurgitation on transvalvular gradient and flow in severe aortic stenosis: a systematic ex vivo analysis of a subentity of lowflow low-gradient aortic stenosis. EuroIntervention. 2018;13: 1635-44.

5. Clavel MA, Burwash IG, Pibarot P. Cardiac imaging for assessing low-gradient severe aortic stenosis. JACC Cardiovasc Imaging. 2017;10:185-202.

6. Clavel MA, Magne J, Pibarot P. Low-gradient aortic stenosis. Eur Heart J. 2016;37:2645-57.

7. Clavel MA, Messika-Zeitoun D, Pibarot P, Aggarwal S, Malouf J, Araoz P, Michelena H, Cueff C, Larose É, Capoulade R, Vahanian A, Enriquez-Sarano M. The complex nature of discordant severe calcified aortic valve disease grading: new insights from combined Doppler echocardiographic and computed tomographic study. J Am Coll Cardiol. 2013;62:2329-38.

8. Benfari G, Clavel MA, Nistri S, Maffeis C, Vassanelli C, Enriquez-Sarano M, Rossi A. Concomitant mitral regurgitation and aortic stenosis: one step further to low-flow preserved ejection fraction aortic stenosis. Eur Heart J Cardiovasc Imaging. 2017 Jul 27. [Epub ahead of print].
9. Baumgartner H, Falk V, Bax JJ, De Bonis M, Hamm C, Holm PJ, Iung B, Lancellotti P, Lansac E, Muñoz DR, Rosenhek R, Sjögren J, Tornos Mas P, Vahanian A, Walther T, Wendler O, Windecker S, Zamorano JL; ESC Scientific Document Group. 2017 ESC/EACTS Guidelines for the management of valvular heart disease. Eur Heart J. 2017;38:2739-91.

10. Barbanti M, Webb JG, Hahn RT, Feldman T, Boone RH, Smith CR, Kodali S, Zajarias A, Thompson CR, Green P, Babaliaros V, Makkar RR, Szeto WY, Douglas PS, McAndrew T, Hueter I, Miller DC, Leon MB. Impact of preoperative moderate/ severe mitral regurgitation on 2-year outcome after transcatheter and surgical aortic valve replacement: insight from the Placement of Aortic Transcatheter Valve (PARTNER) Trial Cohort A. Circulation. 2013;128:2776-84.

11. Nombela-Franco L, Eltchaninoff H, Zahn R, Testa L, Leon MB, Trillo-Nouche R, D'Onofrio A, Smith CR, Webb J, Bleiziffer S, De Chiara B, Gilard M, Tamburino C, Bedogni F, Barbanti M, Salizzoni S, Garcia Del Blanco B, Sabate M, Moreo A, Fernandez C, Ribeiro HB, Amat-Santos I, Urena M, Allende R, Garcia E, Macaya C, Dumont É, Pibarot P, Rodés-Cabau J. Clinical impact and evolution of mitral regurgitation following transcatheter aortic valve replacement: a meta-analysis. Heart. 2015;101:1395-405. 